\title{
Development of Algorithm for Detecting Strong Wind Location due to Gust Using Weather Radar Information
}

\author{
Takaaki FUKUHARA \\ Kazuya TAKAMI \\ Keiji ARAKI \\ Meteorological Disaster Prevention Laboratory, Disaster Prevention Technology Division
}

\begin{abstract}
Since gusts such as tornados, gust fronts, and downbursts are localized and short time phenomena, they cannot always be detected with existing anemometers along railways used for train operation. Therefore, to ensure keep the safety of train operations against these gusts, an algorithm was developed for extracting the strong wind areas on the ground. This algorithm is based consists of two parts. The first is to detect gust indices in upper air layers from echo and Doppler wind velocity observed with existing weather radar, and the second is to estimate the surface wind velocity of ground surface from upper air gust indices using calculation results of previous gust events using a meteorological model.
\end{abstract}

Keywords: gust, weather radar, numerical simulation

\section{Introduction}

Train operation regulations to prevent disasters caused by strong wind on the railways are implemented when the indicated value from an anemometer installed along a railway line exceeds a given threshold. Anemometers along railway lines are installed at an average interval of $15 \mathrm{~km}$ to $20 \mathrm{~km}$. Therefore, strong winds that blow for a long time across a relatively wide area, such as typhoons and low pressure, can be detected. On the other hand, gusts such as tornadoes linked to recent reports of trains overturning cannot be detected. The spatial size of a gusts is $100 \mathrm{~m}$ to 1 $\mathrm{km}$, which is less than an average interval of between anemometers. Consequently, accurately detecting gusts with anemometers is difficult. Even if the installation deployment density of the anemometer was increased to make it possible to detect gusts, there may not be in time to implement the relevant operational regulations when the train is already approaching the corresponding area.

In recent years, various studies using meteorological radar information for detecting and forecasting gusts have been carried out. The Japan Meteorological Agency provides information on the possibility of gusts, such as tornado warning information and nowcasts of tornados. In addition, some railway companies use their own weather radar information to implement operational regulations against gusts effectively.

Railway Technical Research Institute (RTRI) has been conducting research and development on operational control, using information from existing weather radars, to improve the safety of railway operations when gusts occur. Since precipitation and wind information obtained by weather radars is the information of the upper air layer, such of ground information cannot be obtained directly. Even when if tornado warnings information, mentioned above, are published, damage does not always occur on the ground. In such, cases where the wind is weak at on ground even if there are gusts in the upper air, may occur. Numerical simulation is an alternative means to obtain a distribution of wind or rain when the weather conditions cannot be obtained by observation. By calculating the relationship between the wind in the upper air level and wind at ground level when the gust occurs, the state of the gust at ground level that would affect the operation of the railways can be determined based on the information obtained by the radars.

Therefore, we studied an algorithm for estimating the wind velocity at ground level due to gusts blowing along the railway line by combining weather radar information with the wind velocity relationship between the wind in the upper air and on the ground.

\section{Overview of algorithm}

An algorithm was examined using weather radar information (Fig. 1) to extract the locations of 'gust' such as tornadoes, downbursts, and gust fronts, or sudden increases in wind velocity caused by the passage of the front.

This algorithm consists of two parts: first algorithm is using Doppler wind velocity obtained from a weather radar (A of Fig. 1) to detect locations where vortex, divergence and convergence of wind, which are related to tornados, downbursts and gust fronts, are remarkable. The precipitation and wind observed with the weather radar are values found at a height of about several hundred meters to $3 \mathrm{~km}$ above the ground. Therefore, the gust index obtained by the weather radar does not always match the wind conditions on the ground.

Second algorithm estimates the wind velocity on the ground using a relational expression, which are obtained by numerical calculation of past gusts using a meteorological model. In the algorithm, the relational expression comes from the gust index detected from the results of weather radar observations to estimate the location of strong winds (B in Fig. 1).

Finally, to determine whether the gust can subsequently reach the railway lines, after the movement of the extracted strong wind areas are extrapolated using the movement vector of the gust index, the time change of the strong wind areas is predicted.

The detection of the gust index by weather radars is described in Chapter 3, and the estimation of the wind velocity on the ground using the gust index is described in Chapter 4 in detail. 


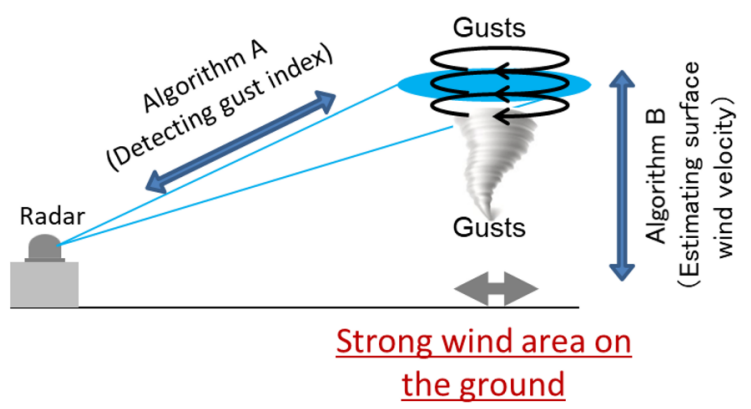

Fig. 1 Algorithm for detecting strong wind area due to gusts

\section{Methods to detect of gust index using weather ra-} dar

When a gust occurs, wind vortices in a tornado, wind divergence in a downburst, and wind convergence in a gust front, stand out in the upper sky. This chapter describes a method to detect these remarkable vortices, divergence, and convergence (gust index) in the sky using a meteorological radar. Since the vortex of the tornado shown above is small on a horizontal scale, it is not directly detected with the weather radar. However, the mesocyclone vortex that produces the tornado can be observed directly with weather radar. Therefore, the tornado is detected by detecting the vortex of mesocyclone.

\subsection{Weather radar information used for analysis}

By using the weather radar, from the reflection of radio waves by precipitation particles, the reflection intensity (the intensity of radio waves reflected by precipitation particles, which is converted into precipitation) and the Doppler velocity (the line of sight of the radar beam) is observed as data in the gaze and azimuth directions (rotational directions on concentric circles) of the radar beam (Fig. 2). In this study, observation data was used from four X-band MP radars (hereafter, XRAIN) provided by the Ministry of Land, Infrastructure, Transport and Tourism located on the side of the Sea of Japan. XRAIN uses two elevation angles to distribute rainfall information (observation of each elevation angle is once every two minutes), and 10 elevation angles to create CAPPI data (Constant Al-

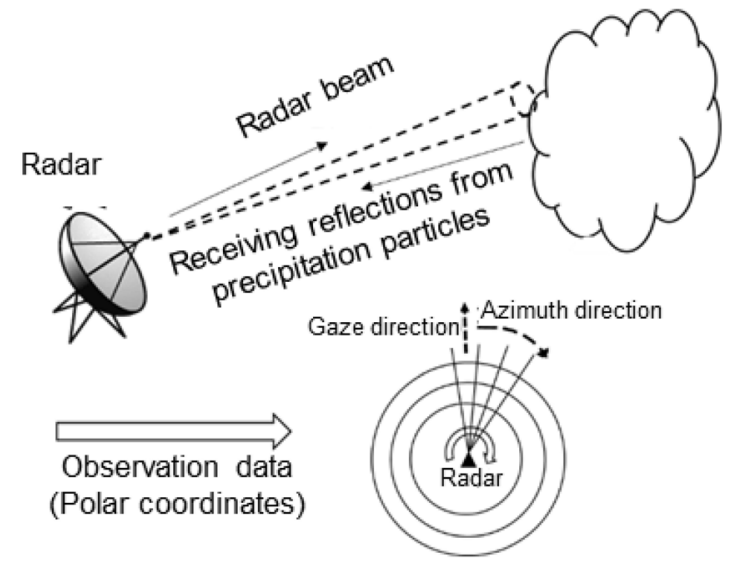

Fig. 2 Conceptual figure of weather radar observation titude Plan Position Indicator, data on iso-altitude planes cut out using multiple elevation angles) (Each elevation is observed once every 5 minutes.)

Hence, a full set of observations are made at 12 elevation angles. In this study about the gust index detection method, three elevation angles were used (one lower elevation angle for rainfall information distribution and the lower two elevation angles for CAPPI data creation).

\subsection{Methods to detect gust index}

Values observed with a weather radar are not the wind velocity distribution itself, but the distribution of the Doppler velocity, which is a one-dimensional velocity of only the line-of-sight component of the radar beam (the direction away from the weather radar is positive). To develop this algorithm, the vortex, divergence, and convergence caused by mesocyclones, downbursts, gust fronts, and fronts, respectively, were obtained from the Doppler velocity distribution obtained from the meteorological radar using the methods developed in previous researches [1] [2] [3]. The detection was performed by paying attention to the increase / decrease area in the gaze direction or the azimuth direction. Figure 3 shows an image of detection with weather radar.

The areas where the Doppler velocity increased or decreased in the direction of the line of sight or the azimuthal direction (solid red line in Fig. 3; hereinafter referred to as the shear segment) were extracted by using the weather radar. This shear segment corresponds with a characteristic wind flow (vortex, wind divergence and wind convergence; shown with white space on colored background arrows in Fig. 3) observed when a gust occurs. Figure 4 shows an extracted example of a shear segment. This figure shows the distribution in the gust front case of Doppler velocity (dotted line) and the smoothed value after the moving average (solid line) of the Doppler velocity in the gaze direction (i.e., distance from radar) at one azimuth.

The area extracted as a shear segment in the gust front and front is the area which is between the maximum $\left(V_{+}\right)$and the minimum $\left(V_{-}\right)$in the gaze direction of the Dop-

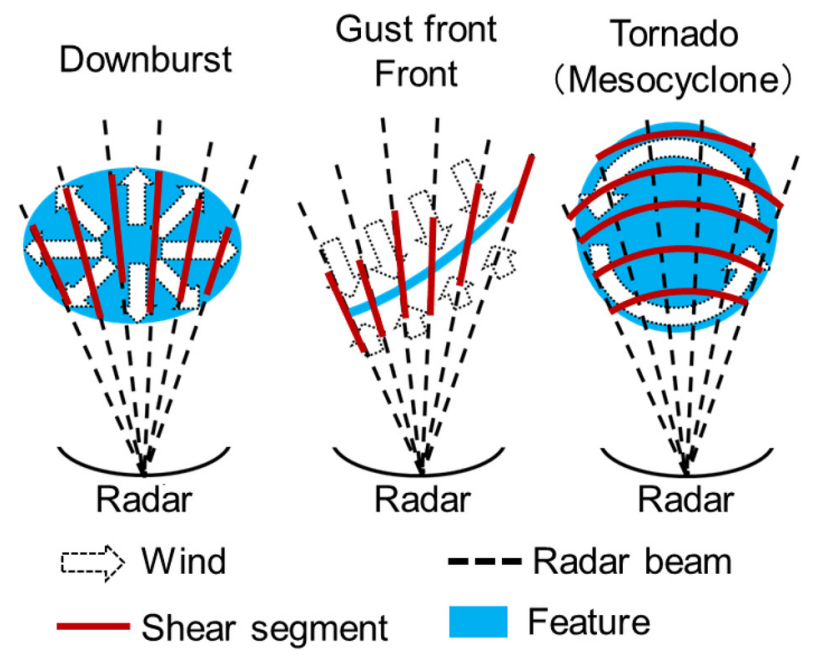

Fig. 3 Conceptual figure of detecting the characteristic wind flow in the sky when a gust occurs using radar 


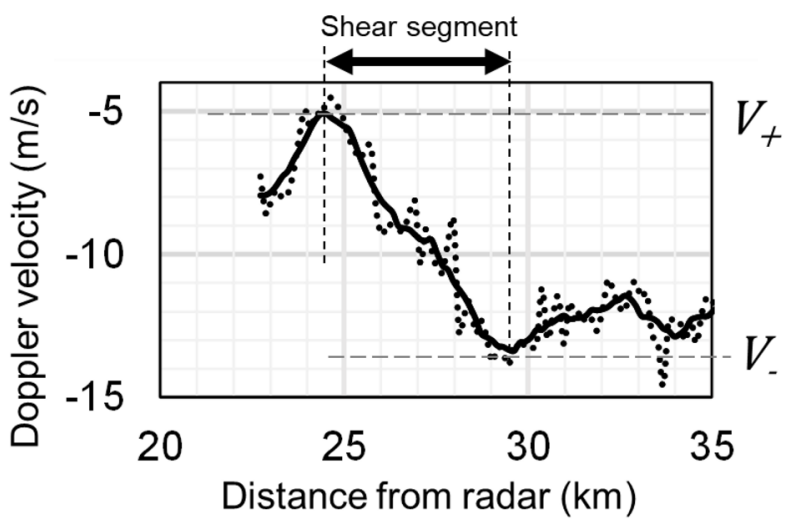

Fig. 4 Example of Doppler velocity extraction for extracting shear segments

pler velocity, which decreases with increasing distance from the weather radar. The area extracted as a shear segment in the downburst, is an area where the Doppler velocity increases in the gaze distribution of the Doppler velocity as distance from the weather radar increases. In the mesocyclone, a region that increases clockwise in the azimuthal distribution of Doppler velocity is extracted.

Vortices, wind divergence and convergence, may be observed in the sky even though when no gust occurs at the ground level. Therefore, to detect the divergence and convergence of the wind and the vortex corresponding to the gust on the ground, a threshold is set for the detection of the shear segment causing the gust. Here, the relative velocity $(V r)$ from the surrounding wind velocity field in the sky, is simplified as shown in (1), using the maximum value $\left(V_{+}\right)$and the minimum value $\left(V_{-}\right)$, shown in Fig. 4.

$$
\begin{aligned}
& V r=\left(V_{+}-V_{-}\right) / 2 \quad \text { (Mesocyclone and downburst) } \\
& V r=V_{+}-V_{-} \quad \text { (Gustfront, and front) }
\end{aligned}
$$

In this algorithm, the threshold value of $V r$ is determined to avoid missing gusts generated in the target area. In setting the threshold, eight cases were used, including cases where gust damage occurred in the past in the target XRAIN observation area or cases where a sudden increase in instantaneous wind velocity was confirmed at the Automated Meteorological Data Acquisition System (hereafter, AMeDAS) observation point of the Japan Meteorological Agency. In each case, the $V r$ at which the shear segment could be detected was examined. The minimum value was $3.0 \mathrm{~m} / \mathrm{s}$ immediately above the spot where damage from gust had occurred, or the AMeDAS observation point. From this, the threshold value of $V r$ for detecting a shear segment with the present algorithm was set to $3.0 \mathrm{~m} / \mathrm{s}$, and those having $V r$ equal to or more than this value were detected as shear segments.

The shear segments extracted in this manner are onedimensional values in the gaze direction or the azimuth direction. Therefore, the same type of adjacent shear segments is integrated and detected as two-dimensional areas (the blue area in Fig. 3; hereinafter, referred to as a feature). In addition, in order to reduce erroneous detection due to the influence of terrain, etc., the feature has to be detected at two or more of the three elevation angles. In this algorithm, the shape of the feature to be detected was approximated with a circle for the mesocyclone, an ellipse for the downburst, and a curve for the gust front and front.

Next, the threshold of the feature detection of the gust index was determined. As a result of examining the Vr of the shear segment in the feature for the eight cases described above, it was found that there is one with a $\mathrm{Vr}$ of 5.0 $\mathrm{m} / \mathrm{s}$ or more. From this, the threshold value of the feature that may generate a gust on the ground was empirically set to $5.0 \mathrm{~m} / \mathrm{s}$ for the maximum value of the shear segments that composed it, and then a value exceeding this value was detected as a gust index.

As a detection example of the gust index set by the above method, an example in which the convergence of the wind accompanying the gust front was detected will be described. Figure 5 shows the Doppler velocity distribution when this example occurred, and Fig. 6 shows the shear

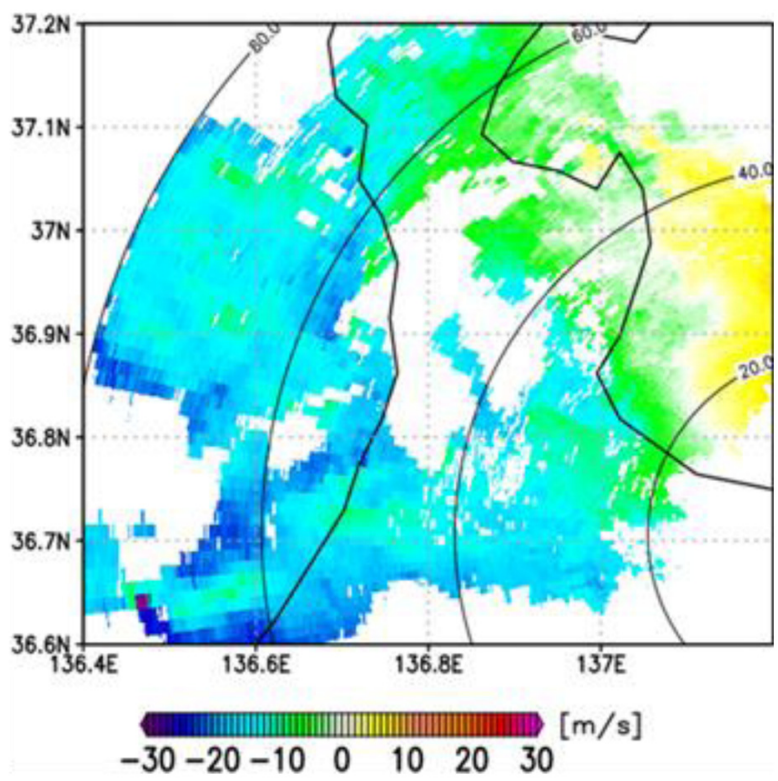

Fig. 5 Example of Doppler velocity distribution

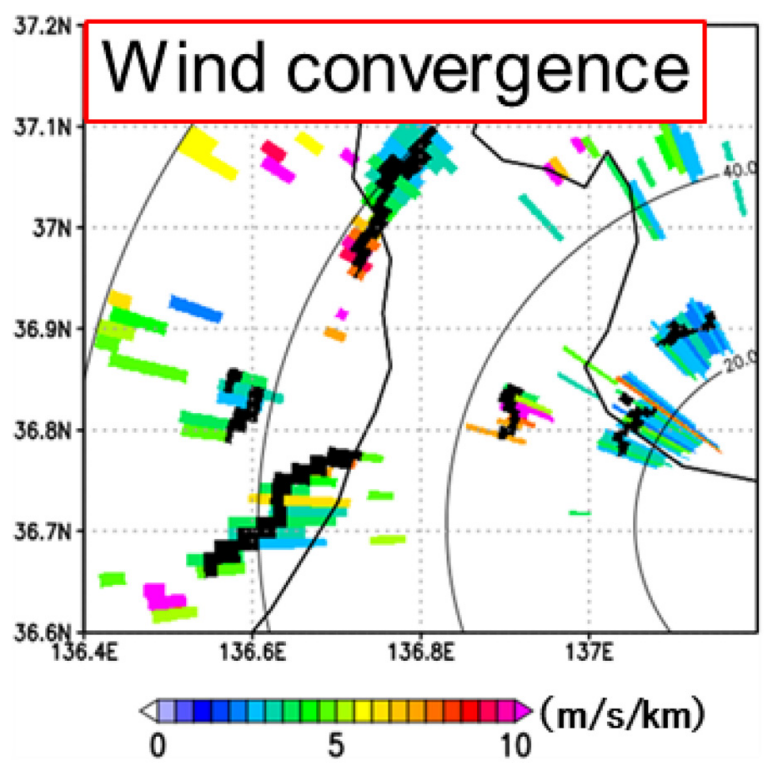

Fig. 6 Example of detecting shear segment and gust index (Gust front, wind convergence) 
segment extracted from this distribution and the detected gust index. Thus, it was found that the gust index can be detected from the weather radar information.

\subsection{Prediction of movement of detected gust index}

To prevent railway disasters, information on the possibility that a gust on the ground can reach tracks and trains is needed. This section describes the prediction of gust index movement.

Gust indices are often detected in rain clouds with strong precipitation, such as cumulonimbi. In the field of precipitation forecasting such as nowcast, kinematic forecasting methods are used for actual operation as a method of forecasting the movement of patches of rain. The kinematic prediction method is a method for predicting movement under the assumption that the precipitation area observed at that time moves along a certain movement vector. Since the position of the gust index may change in the cumulonimbus depending on the observation time, it is easier to track the movement of the rain cloud accompanying it than the movement of the gust index itself. Therefore, it was decided to estimate the movement of the gust index using the movement vector of rain clouds accompanying gusts.

In this study, the cross-correlation method was used to estimate the motion vector. In the cross-correlation method [4], calculating the spatial correlation (shifted spatial correlation) of the observed rainfall distribution and the rainfall distribution at a time before the predetermined time while shifting the position, the motion vector which is difference vector finds the shift position having the highest correlation, and determines the shift position. The difference is estimated as a movement vector. In this algorithm, the area where the maximum value of the reflection intensity in the vertical direction is $20 \mathrm{dbZ}$ or more within the area of $10 \mathrm{~km}$ per side centered on the center point of the detected gust index is targeted. The shifted spatial correlation between the maximum value of the reflection intensity at the time when the gust index was detected and the one observed 5 minutes before was calculated. The location of the strongest spatial correlation was estimated as the position of the gust index 5 minutes ago, and the movement vector was calculated to predict the movement of the gust index.

\section{Estimation of ground surface wind velocity due to gusts}

The wind obtained by the weather radar is the wind in the sky, but trains run on the surface of the ground, hence, it is necessary to obtain the wind on the ground level. Therefore, the vertical structure of the gust was obtained by numerical calculation, and the construction of a relational expression between $V r$ (difference in wind velocity) of the gust index described in Chapter 3 and the difference in wind velocity on the ground was examined. From this result, the wind on the ground is estimated. The weather model divides the target area into grids in the longitudinal, latitudinal, and height directions. To each grid, topographic and land use data at each grid point, and weather data such as wind velocity, rain, and temperature (initial conditions and boundary conditions) are given. Thus, the time change of each meteorological element for each grid is calculated by a computer. In this study, the WRF (Weather Research and Forecasting) model [5] was used for numerical simulation.

\subsection{Overview of numerical calculations}

Seven gusts cases were used in the numerical calculations ( 6 tornados and 1 gust front). These cases are specified within the XRAIN observation range within the period in which the XRAIN data had been recorded, and its type was specified. Since no downburst has been observed within this range, they were excluded from the simulation. In each case, the area around 30 to $60 \mathrm{~km}$ square around the point where the ground damage was reported was used as the numerical calculation range, and grids were set at $200 \mathrm{~m}$ intervals in the east-west and north-south directions. The vertical grid had 60 layers. Initial conditions and boundary conditions were created using the Japan Meteorological Agency's meso-scale objective analysis data, global analysis (Japan region) objective analysis data, and objective analysis data (NCEP-FNL) created by the National Centers for Environmental Prediction (NCEP) in the United States.

\subsection{Numerical calculation results of gust events}

Since the height of the gust index obtained from the weather radar depends on the distance from the radar, height information has to be included in the relational expression for estimating the wind on the ground from the difference in wind velocity with the gust index. In this study, the relational expression for calculating the difference in wind velocity on the ground was determined by integrating the difference in wind velocity between layers having a vertical height difference of $200 \mathrm{~m}$. In addition, gusts have a three-dimensional structure, and a region with a large difference in wind velocity is not always upright in the vertical direction. Therefore, when calculating the wind velocity on the ground from the difference in wind velocity with the gust index, it is necessary to consider not only the above-mentioned wind velocity differences, but also horizontal displacement of that area.

First, a plane with a certain height from the sea surface was set, and the difference in wind velocity between the two points within that surface was determined from the three-dimensional wind direction and wind velocity obtained by numerical calculation (Fig. 7). Since the spatial correlation of the difference in wind velocity obtained at each of the two altitude planes with a height difference of $200 \mathrm{~m}$ has a strong positive correlation, the method of creating the relational expression is considered to be generally valid.

Next, the shift between ground level and at altitude in regions with large differences in wind velocity, were examined. The spatial correlation between the distribution of the wind velocity difference at the height $\mathrm{Z}$ from the sea surface and the distribution of the wind velocity difference at the height $\mathrm{Z}+200 \mathrm{~m}$ was calculated while shifting the center position of the spatial distribution at the height $\mathrm{Z}+$ 


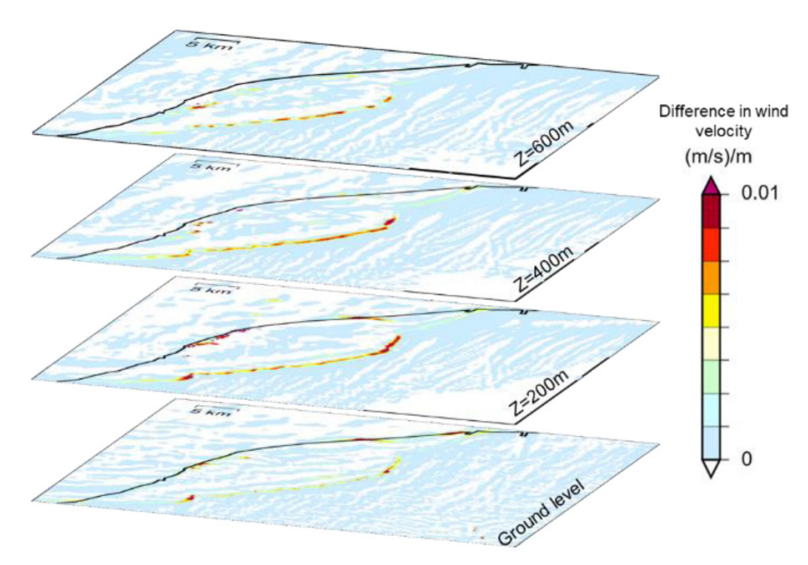

Fig. 7 Calculation example of difference in wind velocity between two points at each altitude plane

$200 \mathrm{~m}$. As a result, it was found that the correlation coefficient was highest when there was no shift in the eastwest and north-south directions. From this fact, it was considered that there is no horizontal displacement of the area where the difference in wind velocity is large in two altitude planes with an altitude difference of $200 \mathrm{~m}$. Based on these results, a relational expression between the difference in wind velocity of the gust index and the difference in wind velocity on the ground was constructed.

The difference in wind velocity between adjacent grid points at a certain altitude was calculated for gust front cases. Figure 8 shows the values obtained by dividing the difference in wind velocity at the altitude plane $\mathrm{Z}$, by the difference in wind velocity at the altitude plane $(\mathrm{Z}-200 \mathrm{~m})$ $200 \mathrm{~m}$ below. If the value is greater than 1, the difference in wind velocity at the higher altitude plane is larger than at the lower altitude plane. On the gust front, the wind velocity difference increased from the ground to a height of $400 \mathrm{~m}$. On the other hand, at points higher than $400 \mathrm{~m}$, the difference in wind velocity tended to decrease.

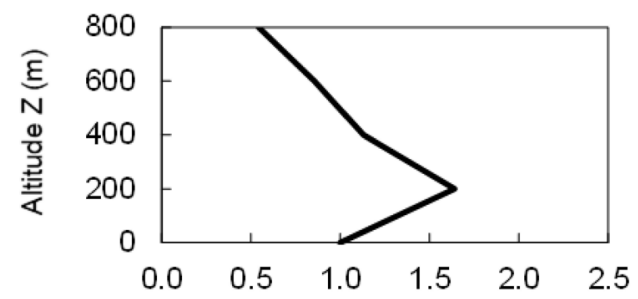

Ratio of difference in wind velocity between two altitude planes Plot: Difference in wind velocity at that height and more
ratio to difference in wind velocity at $200 \mathrm{~m}$ below

Fig. 8 Relationship between wind velocity differences in the sky and wind velocity differences above the ground in the case of a gust front

\subsection{Estimation of ground surface wind velocity due to gusts}

The relational expression of the difference in wind velocity at height $\mathrm{Z}\left(U D_{z}\right.$, equivalent to $\mathrm{Vr}$ in (1)) compared to that at ground level $\left(U D_{s f}\right)$, obtained from the difference in wind velocity between the two altitude planes in section 4.2 ,

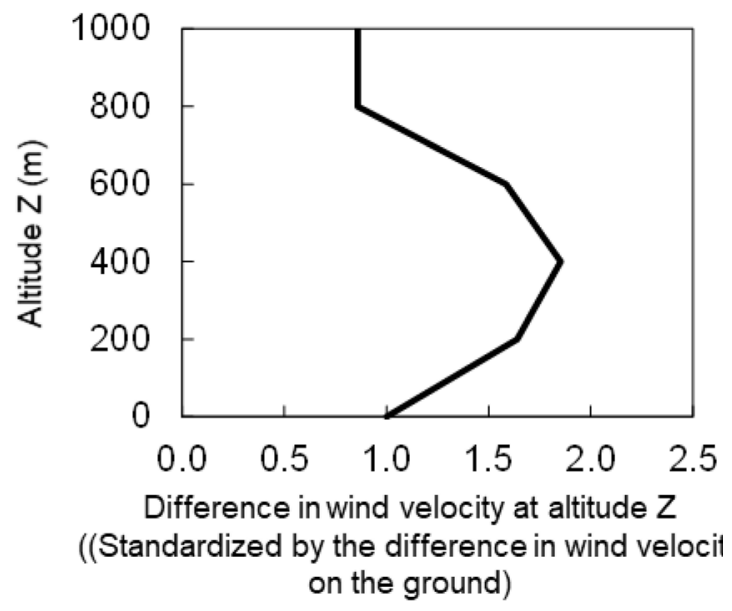

Fig. 9 Difference in wind velocity at altitude $Z$ in the gust front case

is described in this section. The equation for the gust front is shown in (2), and that for the tornado is shown in (3).

Gust front

$U D_{s f c}=\left(Z F\left(z_{d}-800,1.00\right)\right) \times\left(Z F\left(z_{d}-600,1.84\right)\right) \times$

$\left(Z F\left(z_{d}-400,1.17\right)\right) \times\left(Z F\left(z_{d}-200,0.89\right)\right) \times 0.61 \times U D_{z}$

Tornado

$U D_{s f c}=1.0 \times U D_{z}$

Here, the function $Z F(a, b)$ indicates $b$ if $a$ is 0 or more, and 1 if $a$ is less than 0 .

Figure 9 shows the difference in wind velocity at height $\mathrm{Z}$ when the difference in wind velocity on the ground is set to 1 , which is obtained by (2) for the case of a gust front.

\subsection{Example of surface wind velocity estimation}

The wind velocity on the ground is estimated from the wind velocity difference in the gust index described in Chapter 3 and (2) and (3).

For phenomena other than tornadoes, such as gust fronts, the difference in wind velocity $((2)$ in the case of the gust front) on the ground between that obtained by weather radar and by numerical calculation is taken as the difference in wind velocity on the ground.

The wind velocity difference of a tornado described in this chapter is thought to be due to the vortex of the mesocyclone. Then, it is assumed that a tornado is generated in the vortex of the mesocyclone. To convert this into a wind velocity of the tornado, the Rankine vortex model is used, which is widely used in approximating the wind velocity distribution of a tornado. The Rankine vortex model is a model in which the wind velocity increases linearly from the center of the tornado to the maximum tangential wind radius, and the wind velocity is inversely proportional to the distance from the center to the outside. In this algorithm, the radius at the maximum tangential wind velocity (the maximum value of the wind velocity at the Rankine vortex) was set to $50 \mathrm{~m}$, which is the half of the average damage width of the tornado case in Japan (approximately $100 \mathrm{~m}$ ). The maximum tangential wind velocity was determined assuming that the difference in wind velocity on the 
ground obtained by (3) occurred outside the Rankine vortex, and this value was taken as the gust-body wind velocity difference on the ground.

When a gust is generated, the wind is blowing not only in the gust body such as the gust front but also in the vicinity of the gust. The wind velocity at the time of the gust occurrence can be regarded as the sum of the difference between the peripheral wind velocity and the wind velocity due to the gust. Therefore, the wind velocity on the ground is calculated as a value obtained by adding the average wind velocity (wind velocity in the environmental field) observed at a surrounding weather station (such as AMeDAS) to the gust wind velocity difference of on the ground.

In the case of a gust front, the wind velocity difference obtained by numerical calculation is used. It is necessary to convert the obtained value to the instantaneous wind velocity since the wind velocity obtained by numerical calculation is an average value. The ratio of the maximum instantaneous wind velocity to the average wind velocity was calculated based on the observation values obtained at the AMeDAS observatory of the Japan Meteorological Agency in the case of a gust front that occurred in the area where the XRAIN data was analyzed. The ratio was 1.8 on average. In cases other than tornadoes, this value is finally multiplied to obtain the estimated value of the maximum instantaneous wind velocity on the ground.

\section{Analyses and discussions Example of extracting strong wind area by this algorithm}

An example of extracting a gust front strong wind area using the proposed algorithm is described in this chapter.

Figure 10 shows the echo intensity observed at a certain time and the gust index extracted by the method described in Chapter 3. The red solid line in the figure is a gust index corresponding to the gust front. The difference in wind velocity from this gust index, which was calculated from the Doppler wind velocity on a weather radar, was about $6 \mathrm{~m} / \mathrm{s}$. The wind velocity of the environmental field was $5 \mathrm{~m} / \mathrm{s}$ calculated from the average wind velocity

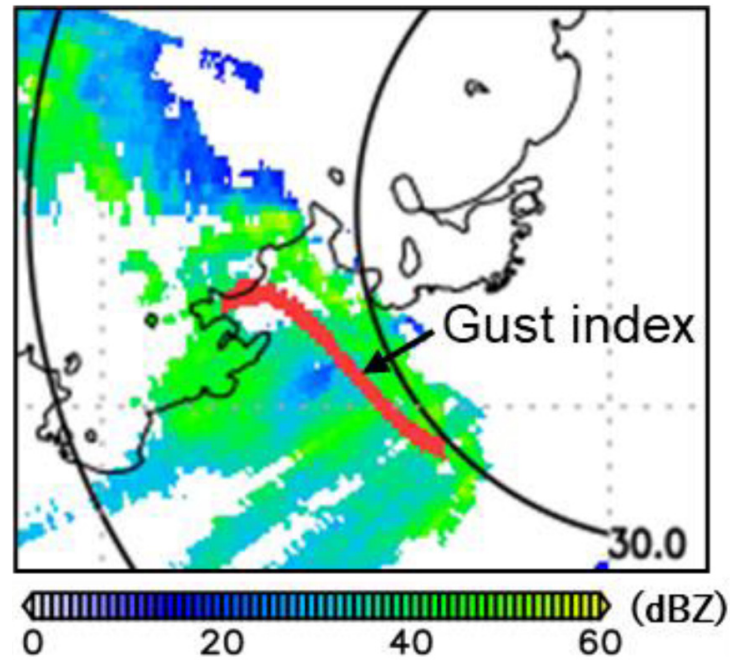

Fig. 10 Observed echo intensity and gust index corresponding to gust front
Table 1 Example of estimated and observed maximum instantaneous wind velocity in the gust front case

\begin{tabular}{c|c}
\hline & $\begin{array}{c}\text { Maximum instantaneous } \\
\text { wind velocity }\end{array}$ \\
\hline $\begin{array}{c}\text { Estimated value } \\
\text { (Using this Algorithm) }\end{array}$ & $21.6 \mathrm{~m} / \mathrm{s}$ \\
\hline Observed value & $21.0 \mathrm{~m} / \mathrm{s}$ \\
\hline
\end{tabular}

at the surrounding AMeDAS stations before and after the time when the gust index was detected. The gust index was calculated using Equation (2), the wind velocity in the environmental field, and the ratio of the maximum instantaneous wind velocity to the average wind velocity, 1.8. The result was $21.6 \mathrm{~m} / \mathrm{s}$ as shown in Table 1 . Therefore, this gust can be estimated to be more than a $20 \mathrm{~m} / \mathrm{s}$ instantaneous wind velocity on the ground.

In contrast, the maximum instantaneous wind velocity observed at the AMeDAS observatory before and after the occurrence of the gust front was $21.0 \mathrm{~m} / \mathrm{s}$. From the result, it is considered that the maximum instantaneous wind velocity on the ground in this case can be roughly estimated by this algorithm.

\section{Conclusions}

Since gusts (tornadoes, downbursts, gust fronts, and rapid increases in wind velocity caused by passing through the front) are local and short-time phenomena, they cannot always be detected accurately with existing anemometers.

Therefore, we constructed an algorithm to detect the area of these strong winds by combining the following two methods and to estimate the movement of the area.

1) Detection method of a gust index that indicates the magnitude of the divergence and convergence of the remarkable sky wind and the strength of the vortex, such as a strong wind due to a gust on the ground, from existing weather radar information.

2) Estimation method of the difference in wind velocity on the ground from the difference in wind velocity in the gust index obtained from numerical calculation of past gusts etc.

As a result, it was found that the proposed algorithm can roughly estimate the strong wind area on the ground due to gusts.

The threshold used for detecting the gust index in the proposed algorithm and the relational expression between the difference in wind velocity on the gust index and the difference in wind velocity on the ground were determined by gusts generated in two areas on the Sea of Japan side. Therefore, so far it has not yet been verified that it can be applied all area of Japan. We plan to verify these and improve the algorithm with the goal of improving its reliability in the future study.

\section{Acknowledgement}

XRAIN data is provided by the Ministry of Land, Infrastructure, Transport and Tourism. The datasets used 
were collected and provided under the Data Integration and Analysis System (DIAS) developed and operated by the Ministry of Education, Culture, Sports, Science and Technology.

\section{References}

[1] Stumpf, G. J., A. Witt, E. D. Mitchell, P. L. Spencer, J. T. Johnson, M. D., Eilts, K. W. Thomas, and D. W. Burgess, "The National Severe Storms Laboratory Mesocyclone Detection Algorithm for the WSR-88D," Wea. Forecasting, 13, pp.304 326, 1998.

[2] Merritt, M.W., "Microburst divergence detection for terminal Doppler weather radar," Preprints, 24th Conf. on Radar Meteor., Tallahassee, FL, Amer. Meteor. Soc., pp.220 223, 1989.

[3] Hermes, L. G., A. Witt, S. D. Smith, D. Klingle-Wilson, D. Morris, G. J. Stumpf, and M. D. Eilts, "The Gust front detection and wind-shift algorithms for the terminal Doppler weather radar system," J. Atmos. Oceanic Technol., 10, pp.693 709, 1993.

[4] Bellon, A., and G. L. Austin, "The evaluation of two years of real-time operation of a short-term precipitation forecasting procedure (SHARP)," J. Appl. Meteor., 17, pp.1778 1787, 1978 .

[5] W. C. Skamarock, J. B. Klemp, J. Dudhia, D. O. Gill, D. M. Barker, M. G. Duda, X.-Y. Huang, W. Wang, J. G. Powers, "A Description of the Advanced Research WRF Version 3," NCAR Technical Note, 2008.

\section{Authors}

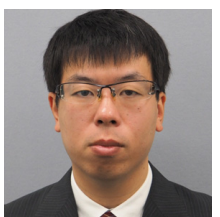

Takaaki FUKUHARA

Senior Researcher, Meteorological Disaster Prevention Laboratory, Disaster Prevention Technology Division

Research Areas: Strong Wind Disasters

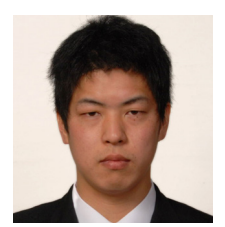

Kazuya TAKAMI

Researcher, Meteorological Disaster

Prevention Laboratory, Disaster Prevention Technology Division

Research Areas: Strong Wind Disasters

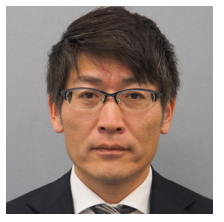

Keiji ARAKI

Chief Researcher, Meteorological Disaster Prevention Laboratory, Disaster Prevention Technology Division Research Areas: Strong Wind Disasters 